\title{
CARACTERIZACIÓN CUÁNTICA DE MATERIALES MEDIANTE LA TEORÍA DE LA FUNCIONAL DENSIDAD
}

\author{
(Quantum characterization of materials using the density functional theory)
}

\section{Carlos Alberto Uribe Suárez*, Ricardo Hernández Ramos**}

\author{
*Escuela de Ingeniería de Minas, UPTC. Grupo de investigación GEAM, geam@yahoo.com . \\ **Escuela de Ingeniería Geológica, UPTC. Grupo de investigación GEAM, geam@yahoo.com .
}

(Recibido: el 1 de Abril de 2014 y aceptado 1 de Octubre de 2014)

\begin{abstract}
Resumen:
La caracterización teórica de materiales, a la luz de la mecánica cuántica, revela aspectos completamente novedosos. Su implementación requiere el conocimiento previo de su estructura cristalina, los átomos que la componen y sus posiciones en ella (primeros principios). Al resolver la ecuación de Schrodinger, es posible conocer la energía del sistema cristalino y con ella el valor del parámetro de red que la minimiza. Con estos se determinan los valores de energía en diferentes direcciones de primera zona de Brillouin o estructura de bandas y, finalmente, esta información conduce a la caracterización mecánica, eléctrica y óptica del material.
\end{abstract}

Palabras clave: DFT, LAPW, estructura de bandas, función dieléctrica, primeros principios.

\section{INTRODUCCIÓN}

En la actualidad es viable realizar estudios de las propiedades de diversos materiales mediante métodos de primeros principios, debido a que se ha avanzado mucho en la utilización de herramientas de cómputo, soportadas en un riguroso formalismo teórico. Un método es de primeros principios (de acuerdo con los parámetros de entrada), cuando se tienen en cuenta solamente datos estructurales del material para proveer resultados acerca de sus propiedades electrónicas, a diferencia de los métodos empíricos o semiempíricos, los cuales utilizan resultados previos de un método de primeros principios para producir nuevos resultados. Una característica común de los métodos de primeros principios es la elevada exigencia en capacidad de cómputo, la cual se deriva de los procesos autoconsistentes que se requieren para la obtención de los resultados.

Gran parte de los cálculos de primeros principios para solidos están basados en la teoría de la funcional densidad (Density Functional Theory DFT), la cual se aplica tanto para átomos como para moléculas. En este formalismo, los orbitales electrónicos son soluciones a una ecuación tipo Schrodinger, la cual depende de la densidad electrónica, más que de los orbitales de electrón individuales.

\section{Abstract:}

Theoretical characterization of materials, from quantum mechanics, reveals entirely novel aspects. Its implementation requires prior knowledge of its crystalline structure, the atoms that compose it and their positions in it (First Principles). Solving the Schrodinger's equation is possible to know the energy of the crystalline system and with it the value of the parameter of the network that minimizes it. With these are determined the values of energy in different directions of first Brillouin zone or band structure, finally this information leads to the mechanical, electrical and optical characterization of material

Keywords: DFT, LAPW, band structure, dielectric function, first principles.

El nitruro de zirconio es un metal de alta dureza y resistente a la corrosión, por lo cual se le utiliza para hacer recubrimientos con películas delgadas sobre superficies de dispositivos tales como brocas y otros elementos que se requieren en trabajos de alto desgaste, utilizando técnicas como evaporación y sputtering.

\section{PROPIEDADES ESTRUCTURALES DE ZrN}

El nitruro de zirconio ( $\mathrm{ZrN}$ ) forma su estructura cristalina como una red cúbica centrada en las caras con motivo diatómico, con los átomos de zirconio en las posiciones reticulares y en el centro de las caras, como se observa en la Figura 1.

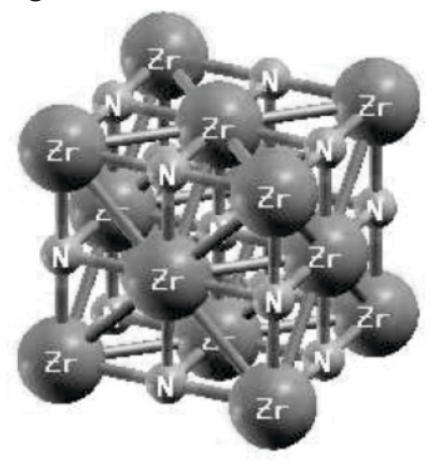

Figura 1. Estructura cristalina de $\mathrm{ZrN}$
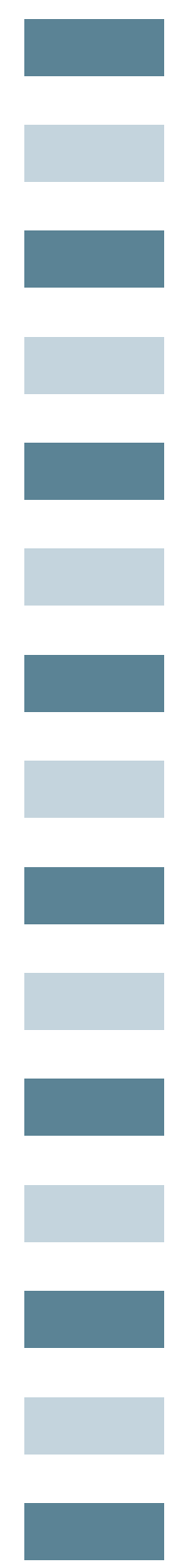
Calculando la energía total para valores arbitrarios y sucesivos del parámetro de red, se obtiene una gráfica energía volumen. El parámetro de red para la mínima energía es de 8.68 Bohr o 4.60 Å con energía de cohesión en el estado base de $-15.13 \mathrm{eV}$. Los valores experimentales reportados para la constante de red están entre $4.56 \AA-4.63$ $\AA$, y $4.61 \AA$ figura 2 .

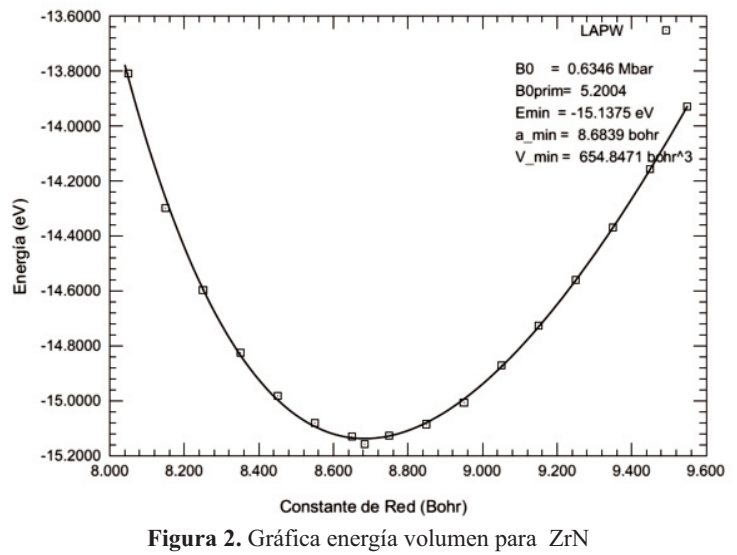

\section{PROPIEDADES ELECTRÓNICAS}

La ecuación de Schroedinger asociada a la fase cristalina, contiene términos correspondientes a la energía cinética de electrones y núcleos, a las interacciones electrón-electrón, núcleo-electrón y núcleo-núcleo. Para el cálculo a primeros principios se utiliza la aproximación de Born-Oppenheimer, la cual considera los núcleos fijos, con el argumento de su mayor masa respecto de la de los electrones, de manera que este potencial resulta constante y la ecuación de Schoerdinger se reduce a:

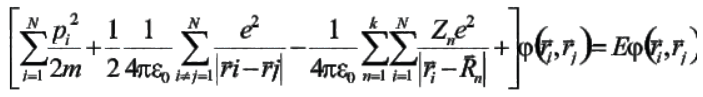

Para solucionar esta ecuación se recurre a la Density Functional Therory (DFT), en la cual se tiene una funcional de energía que depende de la densidad electrónica del estado base, que, a su vez, depende únicamente de tres coordenadas espaciales. La funcional densidad se minimiza mediante el método de los multiplicadores de Lagrange, con la restricción de que la función de onda esté normalizada.

Utilizando una base de funciones de onda apropiadas (armónicos esféricos y ondas planas), se construye la función de onda del sistema y con ella la densidad electrónica.

$$
n(\vec{r})=\sum \varphi_{i}^{*}(\vec{r}) \varphi_{i}(\vec{r})
$$

Al solucionar la ecuación de Poisson con esta densidad se obtiene el potencial de intercambio y correlación.

El problema de muchos electrones interactuantes, se reduce a muchos problemas de electrones no interactuantes en presencia de un potencial efectivo que los involucra.
Con el potencial efectivo se soluciona la ecuación de Schoerdinger para obtener una nueva función de onda del sistema, con ella se recalcula la densidad electrónica y el potencial efectivo. Este proceso se repite hasta alcanzar la convergencia, para un valor de tolerancia dado ("proceso autoconsistente").

Con el potencial efectivo se soluciona la ecuación de Schoerdinger para obtener una nueva función de onda del sistema, con ella se recalcula la densidad electrónica y el potencial efectivo. Este proceso se repite hasta alcanzar la convergencia, para un valor de tolerancia dado ("proceso autoconsistente").

Alcanzada la autoconsistencia, se calculan los valores de energía para diferentes direcciones en el espacio $\mathrm{K}$ o de vectores de onda. Estos resultados en conjunto constituyen la estructura de bandas para el material estudiado, $\mathrm{ZrN}$. Figura 3.

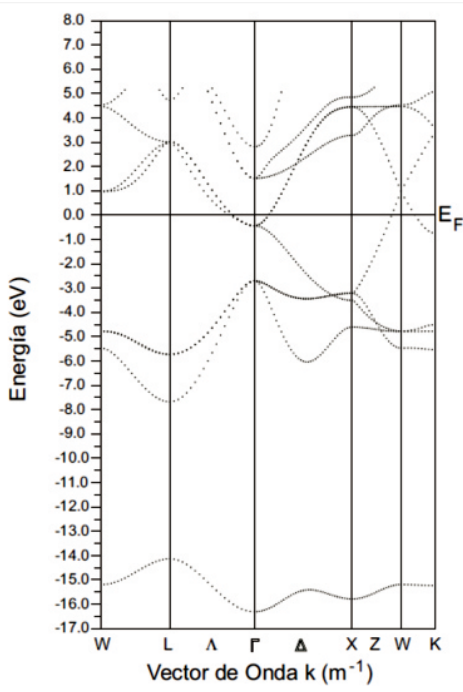

Figura 3. Bandas de energía para $\mathrm{ZrN}$

Esta figura muestra los valores permitidos para la energía de los electrones en diferentes direcciones del espacio recíproco, es análoga a los niveles de energía en un átomo, pero estos son a escala molecular.

La línea continua representa el nivel de Fermi. Las bandas por debajo de este se conocen como de valencia y las que se encuentran por encima, de conducción. Las bandas de valencia se unen en algunos puntos con las de conducción, lo que significa que electrones de valencia pueden migrar a bandas de conducción con muy poca energía adicional. En otras palabras, el material es conductor.

Si se presenta un gap o región prohibida entre las bandas de valencia y conducción, revelaría su carácter dieléctrico.

Dado que los electrones en el cristal solo pueden tomar valores discretos de energía, estos determinan la manera como se absorbe o emite radiación y de aquí sus propiedades ópticas. 
Las propiedades que bajo la mirada de la mecánica clásica parecían constantes, la resistividad, la constante dieléctrica, el índice de refracción, el coeficiente de atenuación y otros, resultan ser funciones de la energía y sus valores cambian en diferentes direcciones cristalinas.

Surge entonces una gran variedad de aplicaciones, dispositivos capaces de detectar pequeños cambios en la orientación del cristal, acelerómetros, dispositivos semiconductores de alta temperatura, herramientas que mientras desarrollan trabajos específicos simultáneamente envían información, emisores muy eficientes y económicos de luz y muchas otras.

\section{CONCLUSIONES}

El gran desarrollo de dispositivos y equipos de cómputo al servicio de la ciencia, ha permitido transformar nuestra visión del universo y con ello la manera como valoramos y utilizamos nuestros recursos naturales.

La ecuación fundamental de la mecánica cuántica para la fase cristalina, puede ser solucionada utilizando algunas aproximaciones $\mathrm{y}$, en particular, la teoría de la funcional densidad.

La cuantización de la energía no es una propiedad exclusiva de los electrones en los átomos, éstos en un cristal solo pueden tomar valores discretos de energía en diferentes direcciones espaciales. Esta relación de dispersión genera la estructura de bandas característica del material.
En general, las propiedades eléctricas, ópticas y térmicas de los materiales se ven afectadas por esta relación de dispersión. Su análisis sugiere múltiples y novedosas aplicaciones que están cambiando y transformaran el mundo con nuevas tecnologías.

\section{REFERENCIAS}

Burke; K. and friends. (2003). The ABC of DFT. New Jersey: Department of Chemistry, Rutgers University.

Nussbaum, A. (1975). Teoría de grupos aplicada para químicos, fisicos e ingenieros.

Thijssen, J.M. (2007). Computational Physics. (2 ${ }^{\text {nd }}$ Ed.). Cambridge University Press.

Uribe, C. \& Rodríguez, J. (2006). Diseño de un método teórico para ajuste de parámetros tight-binding en nitruros. Revista colombiana de física, 38(1). 